\title{
Guillain-Barre Syndrome Following Seasonal Influenza Vaccination
}

\author{
Jason Kei Chak Mak
}

\begin{abstract}
A 77-year-old man with a background of intracranial hemorrhage and hemophilic arthropathy presented with progressive weakness of all four limbs. His symptoms started acutely 8 days after a seasonal influenza vaccination. Physical examination revealed symmetrical proximal muscle weakness and generalized areflexia. Common infectious triggers of Guillain-Barre syndrome (GBS) were ruled out. Neurophysiological studies demonstrated features consistent with GBS. The patient's hospital stay was extensive and complicated by various comorbidities. Despite intravenous immunoglobulin (IVIG) treatment, the symptoms progressed to involve respiratory muscles and the patient was admitted to the intensive care unit. The patient's condition gradually improved after a further course of IVIG, and he was discharged 15 weeks post-admission with ongoing community rehabilitation. The temporal relationship between influenza vaccination and GBS development in this case was suggestive of a vaccineinduced cause, and the clinical implications of this association warrant further research.
\end{abstract}

Keywords: Guillain-Barre syndrome; Autoimmune; Polyradiculopathy; Vaccination; Influenza; Seasonal

\section{Introduction}

Thought to be an autoimmune disorder, Guillain-Barre syndrome (GBS) is an acute, rapidly progressing polyradiculoneuropathy that causes inflammation and demyelination of predominantly motor neurons, resulting in classically symmetrical and ascending muscle weakness, often associated with hyporeflexia or areflexia [1]. Although a rare condition (estimated 1 - 2 cases per 100,000 person-years worldwide [2]), GBS represents the most common cause of acute nontrauma-related paralysis in the developed world. The severity of weakness varies and death can result in severe cases. In approximately two-thirds of patients with GBS, an episode of

Manuscript submitted October 1, 2017, accepted October 18, 2017

Department of Neurology, Queen Elizabeth Hospital Birmingham, Mindelsohn Way, Edgbaston, Birmingham, B15 2GW, UK.

Email: jason.mak@hotmail.co.uk

doi: https://doi.org/10.14740/jnr456e acute infection precedes the neurological symptoms by $1-3$ weeks [3]. Infectious organisms recognized as potential triggers are diverse and include Campylobacter jejuni, cytomegalovirus, influenza viruses, herpes simplex virus (HSV), and human immunodeficiency virus (HIV).

Additionally, there is some evidence to suggest a temporal relationship between GBS and the receipt of various vaccines, including those for rabies, hepatitis (Hep) A and B, polio, and influenza [4]. Such cases are infrequent and the establishment of causality in the majority of these cases has proved controversial; however, a small increased risk of developing GBS specifically after influenza vaccination is relatively well established [5].

\section{Case Report}

\section{History}

A 77-year-old man presented with progressive bilateral upper and lower limb weakness, in addition to mobility problems and feeling generally unwell. The patient's symptoms began acutely 8 days after receiving a seasonal influenza vaccine. There was no prior history of influenza vaccination. The patient denied any headaches, visual disturbances, limb numbness or paraesthesia, or urinary/bowel incontinence. He maintained that he had been well in himself prior to vaccination, and denied any infections in the preceding $2-3$ months. The patient had been fully independent and mobile prior to admission, and there was no history of falls. There was no recent travel history and sexual history was unremarkable.

However, the patient had a significant past medical history. He had severe hemophilia A (hereditary factor VIII deficiency), for which he was on weekly factor VIII prophylaxis (Refacto $\mathrm{AF}$ ), and suffered regular recurrences of generalized arthralgia. $\mathrm{He}$ also had a hemorrhagic stroke 3 years earlier. In addition, the patient was a chronic Hep C carrier with a sustained virologic response (SVR) achieved 8 months prior to admission. The patient's family history was unremarkable. He was a nonsmoker and worked as a bus driver prior to retirement.

\section{Clinical examination}

The patient was initially admitted under hematology and was given tranexamic acid $1 \mathrm{~g}$ PO TDS. He was subsequently re- 
Table 1. Neurological Examination Findings on Admission

\begin{tabular}{|c|c|c|c|c|}
\hline & \multicolumn{2}{|c|}{ Upper limb } & \multicolumn{2}{|c|}{ Lower limb } \\
\hline & Right & Left & Right & Left \\
\hline Appearance & \multicolumn{4}{|c|}{ Normal } \\
\hline Tone & \multicolumn{4}{|c|}{ Normal } \\
\hline \multirow[t]{10}{*}{ Power (Medical Research Council grading system) } & \multicolumn{2}{|c|}{ Shoulder abduction } & \multicolumn{2}{|c|}{ Hip flexion } \\
\hline & $1 / 5$ & $1 / 5$ & $2 / 5$ & $2 / 5$ \\
\hline & \multicolumn{2}{|c|}{ Elbow flexion } & \multicolumn{2}{|c|}{ Hip extension } \\
\hline & $3 / 5$ & $3 / 5$ & $4-/ 5$ & $4 / 5$ \\
\hline & \multicolumn{2}{|c|}{ Elbow extension } & \multicolumn{2}{|c|}{ Knee flexion } \\
\hline & $2 / 5$ & $2 / 5$ & $1 / 5$ & $1 / 5$ \\
\hline & \multicolumn{2}{|c|}{ Wrist extension } & \multicolumn{2}{|c|}{ Knee extension } \\
\hline & $4-/ 5$ & $4-/ 5$ & $2 / 5$ & $2 / 5$ \\
\hline & \multicolumn{2}{|c|}{ Finger adduction } & \multicolumn{2}{|c|}{ Ankle dorsiflexion } \\
\hline & $3 / 5$ & $3 / 5$ & $4+/ 5$ & $4+/ 5$ \\
\hline Reflexes & \multicolumn{4}{|l|}{ Absent } \\
\hline Babinski sign & & & \multicolumn{2}{|c|}{ Negative } \\
\hline Sensation & \multicolumn{4}{|c|}{ Variable and patchy loss to pinprick } \\
\hline Proprioception & \multicolumn{4}{|c|}{ Normal } \\
\hline Coordination & \multicolumn{4}{|c|}{ Unable to assess } \\
\hline
\end{tabular}

ferred to neurology and was assessed with the following examination findings. The patient was alert and oriented to person, place and time (Glasgow coma scale score 15/15). Vital signs were within normal ranges and the patient was apyrexial. There were no signs of head or vertebral trauma. The patient was found to have unequal pupils. A permanent mydriasis in his right eye was noted, caused by trauma during childhood, while right homonymous hemianopia was also noted in the same eye as a result of his previous intracerebral hemorrhage. Visual acuity for the right eye was $6 / 18$, compared to $6 / 6$ for the left. The rest of the cranial examination was unremarkable and no speech abnormalities were identified. Neurological examination revealed symmetrical proximal muscle weakness in both upper and lower limbs, in addition to generalized areflexia. There was variable and patchy loss of sensation to pinprick in all four limbs, but no "glove and stocking" distribution or sensory level was noted. Tone was normal with no extrapyramidal features, and proprioception was preserved in both upper and lower limbs. The Babinski sign was absent. Cardiovascular, respiratory, and abdominal examinations were all unremarkable. These examination findings are summarized in Table 1.

\section{Investigations}

The patient was investigated thoroughly. A head computed tomography (CT) scan with contrast carried out on admission revealed marked focal encephalomalacia in the parietal and occipital lobes caused by a previous intracerebral hematoma, but was negative for any intra/extra-axial hemorrhage or in- tracranial masses. A repeat head CT scan performed several days post-admission confirmed an absence of intracranial pathology. After careful consideration of the patient's past medical history, it was decided that a lumbar puncture would be withheld to prevent any complications secondary to his severe hemophilia.

Nerve conduction studies (NCS) demonstrated absent sensory conduction and decreased motor conduction velocities with dispersed waveforms in both upper and lower limbs, consistent with an acute inflammatory demyelinating polyneuropathy (AIDP). The results are summarized in Table 2. The rest of the neurological workup included an electroencephalogram showing an absence of sub-clinical seizure activity, and an electromyogram (EMG) which revealed a reduced pattern of recruitment in all muscles tested, suggesting a neurogenic pathology. A spine magnetic resonance imaging (MRI) scan revealed spondylotic changes at the lumbar level but no significant thecal or neural compression.

Blood count on admission was unremarkable, with a normal differential, including normal liver function results. Creactive protein level was raised at $136 \mathrm{mg} / \mathrm{L}$ on admission but dropped to $47 \mathrm{mg} / \mathrm{L}$ the following day. Panels for anti-nuclear and antineutrophil cytoplasmic antibodies, as well as a paraneoplastic screen, were all negative. Antiganglioside antibody testing demonstrated positivity for anti-GM1 and anti-GD1b antibodies. Muscle enzyme assays excluded any myopathies. Serum infection screens ruled out any recent infections with Campylobacter jejuni, cytomegalovirus, Epstein-Barr virus, HSV, as well as syphilis or HIV. The patient was immune to Hep A and negative for Hep B. His Hep C viral load was found to be $<12 \mathrm{IU} / \mathrm{mL}$, in keeping with the eradication status prior 
Table 2. Sensory and Motor Nerve Conduction Study Findings on Admission

\begin{tabular}{|c|c|c|c|c|}
\hline Sensory nerve conduction studies & $\begin{array}{l}\text { Peak latency }(\mathrm{m} / \mathrm{s}) \\
\text { (reference value) }\end{array}$ & $\begin{array}{l}\text { Amplitude }(\mu \mathrm{V}) \\
\text { (reference value) }\end{array}$ & $\begin{array}{l}\text { Conduction velocity }(\mathrm{m} / \mathrm{s}) \\
\text { (reference value) }\end{array}$ & \\
\hline \multicolumn{5}{|l|}{ Left median } \\
\hline Index finger - wrist & Absent & - & - & \\
\hline \multicolumn{5}{|l|}{ Left ulnar } \\
\hline Lower leg - ankle & Absent & - & - & \\
\hline \multicolumn{5}{|l|}{ Right superficial peroneal } \\
\hline Lower leg - ankle & Absent & - & - & \\
\hline \multicolumn{5}{|l|}{ Left radial } \\
\hline \multicolumn{5}{|l|}{ Left sural } \\
\hline Motor nerve conduction studies & $\begin{array}{l}\text { Onset latency }(\mathrm{m} / \mathrm{s}) \\
\text { (reference value) }\end{array}$ & $\begin{array}{l}\text { Amplitude }(\mathrm{mV}) \\
\text { (reference value) }\end{array}$ & $\begin{array}{l}\text { Conduction velocity }(\mathrm{m} / \mathrm{s}) \\
\text { (reference value) }\end{array}$ & $\begin{array}{l}\text { F wave }(\mathrm{m} / \mathrm{s}) \\
\text { (reference value) }\end{array}$ \\
\hline \multicolumn{5}{|l|}{ Left median } \\
\hline Wrist - abductor pollicis brevis & $3.70(\leq 4.4)$ & $1.48(\geq 4)$ & $47.5(\geq 49)$ & $31.8(\leq 28.5)$ \\
\hline Elbow - wrist & $9.49(\leq 4.4)$ & $1.08(\geq 4)$ & & \\
\hline \multicolumn{5}{|l|}{ Left peroneal } \\
\hline Ankle - extensor digitorum brevis & $4.59(\leq 6.1)$ & $3.4(\geq 2)$ & $32.1(\geq 41)$ & Not measured \\
\hline Knee - ankle & $16.1(\leq 6.1)$ & $2.1(\geq 2)$ & & \\
\hline Ankle - abductor hallucis & $4.09(\leq 6.1)$ & $10.4(\geq 3)$ & Not calculated & Obscured by muscle artefact \\
\hline \multicolumn{5}{|l|}{ Left ulnar } \\
\hline Wrist - abductor digiti minimi & $2.45(\leq 3.5)$ & $5.9(\geq 6)$ & $37.9(\geq 49)$ & Absent \\
\hline Elbow - wrist & $9.04(\leq 3.5)$ & $2.0(\geq 6)$ & & \\
\hline
\end{tabular}

to admission.

\section{Differential diagnosis}

On initial presentation, the differential diagnosis for an elderly man presenting with bilateral proximal muscle weakness, absent reflexes, and difficulty walking included stroke, intracranial mass, transverse myelitis, multiple sclerosis (MS), AIDP, idiopathic inflammatory myopathies, neuromuscular junction disorders, and botulism.

In light of the patient's past medical history, a hemor- rhagic stroke was an important differential to consider, but the head CT scan on admission excluded any cerebrovascular accidents, and was also negative for intracranial masses. The absence of a sensory level or urinary retention made a myelopathy unlikely, as confirmed by a normal MRI spine. MS is also a demyelinating condition but tends to cause unilateral symptoms, and presents with upper motor neuron signs such as a positive Babinski response. Proximal muscle weakness is highly suggestive of idiopathic inflammatory myopathies such as polymyositis, but the latter would not present with areflexia; also, creatinine kinase levels were normal. Myasthenia gravis can also present with proximal weakness, but this correlates 
with physical activity, and extraocular and/or bulbar musculature is often involved. Ultimately, EMG helped rule out both myositis and neuromuscular junction disorders. Furthermore, from the patient's history, there were no recent behaviors suggestive of botulism and his left pupil had an intact pupillary light response. Overall, the clinical picture combined with the neurophysiological findings was consistent with an AIDP.

In addition to the recent seasonal influenza vaccination, it was important to consider alternative triggers of the GBS in this case. In particular, a recent infection such as Campylobacter jejuni or influenza was statistically the most likely, but the patient denied a recent history of illness and clinical signs supporting active infection on admission were absent. Biochemical tests ruled out several potential infectious organisms that could have triggered the development of GBS in this patient. This included Hep C, of which the patient was a known chronic carrier. Although an extremely rare cause of GBS, a potential association of GBS development with Hep C infection had previously been reported [6]. However, the risk of a late relapse post-SVR is extremely low and the undetectable Hep C viral load in this patient confirmed the successful eradication of the virus.

\section{Treatment}

The patient received two 5-day courses of $30 \mathrm{~g}$ intravenous immunoglobulin (IVIG; Privigen). Additionally, the patient underwent extensive neurological rehabilitation including physiotherapy and speech and language therapy during his hospital stay.

\section{Outcome and follow-up}

The patient's muscle weakness rapidly progressed; he became bed-bound and unable to carry out activities of daily living within 1 week of admission, and required nasogastric feeding. Despite IVIG treatment, his symptoms continued to deteriorate. There was an acute decline of lung function 5 weeks post-admission, with a forced vital capacity of $0.95 \mathrm{~L}$, and the patient appeared distressed despite a normal arterial blood gas. A CT pulmonary angiogram was negative for pulmonary embolism. He was admitted to the intensive care unit for 6 days for non-invasive ventilation. The patient's condition was complicated by the development of hospital-acquired pneumonia, which was treated with co-amoxiclav followed by tazocin, as well as frank hematuria and acute kidney injury, which required urology input and urinary catheterization. Additionally, multiple factor VIII infusions were necessary in view of an acute hemathrosis of the patient's left elbow and recurrent episodes of epistaxis. The patient was also found to be intermittently confused.

The patient's symptoms gradually improved after a second course of IVIG. A repeat set of NCS demonstrated a general improvement in both sensory and motor function. The patient was discharged 15 weeks after admission and was supported by community rehabilitation services post-discharge.

\section{Discussion}

GBS is generally regarded as an autoimmune AIDP and has been hypothesized to result from cross-reactive antigenic stimulation in the host peripheral nervous system. Numerous vaccines, including both seasonal and pandemic influenza vaccines, have been shown to precede the development of GBS; however, whether this relationship is temporal or causal remains controversial.

A potential association between GBS development and influenza vaccination first came to light during the 1976 swine influenza vaccination campaign, when a seven- to eight-fold increased relative risk of GBS in adults was observed in the first 6 weeks post-vaccination [5]. While biologically plausible, the mechanism by which influenza vaccination may provoke new onset and recurrent GBS remains unclear. Moreover, considering the rarity of GBS itself, identifying a small increased risk after vaccination can be extremely difficult. The Institute of Medicine stated in 2011 that "an association cannot be confidently ruled out, particularly for future vaccine strains" [7]. Indeed, several cases describing a possible association have previously been reported $[8,9]$, and their conclusions are consistent with a number of recent studies [10-12] and meta-analysis [13], which suggest the presence of a very small but quantifiable attributable risk (approximately $1-2$ excess cases per one million vaccinated individuals).

The identification and understanding of this risk is important as while the clinical manifestations of GBS are often reversible, they can be serious and result in permanent nerve damage. Additionally, GBS remains a leading cause of acute non-traumatic paralysis, with approximately $30 \%$ of patients developing respiratory failure that require ICU intervention and invasive mechanical ventilation. Furthermore, an early diagnosis of GBS can be complicated by a number of known atypical presentations, in addition to the evolving nature of the influenza vaccine contents both geographically and over time.

However, it must be emphasized that the seasonal influenza vaccine has an excellent safety profile and the risk of developing GBS after receiving this vaccine remains substantially lower than that from acquiring influenza itself. It is extremely important that patients understand both the benefits and potential risks surrounding seasonal influenza vaccination, particularly those over the age of 65 , in whom influenza vaccine efficacy is reduced compared to other age groups.

\section{Conclusion}

In conclusion, the present case highlights a presentation of GBS that had a clear temporal association with a preceding seasonal influenza vaccination. In the vast majority of cases, the risk of developing GBS post-vaccination is outweighed by the established benefits of vaccination, but careful active surveillance of an increased GBS incidence during seasonal (and pandemic) influenza vaccination campaigns is clearly warranted. Future research should aim to determine the predisposing host factors and biological mechanisms underlying this potential association. 


\section{Acknowledgments}

The author would like to thank Dr A. P. Mocroft for providing the results of the nerve conduction studies.

\section{Funding Support}

The author did not receive any funding for this work.

\section{Conflict of Interest}

The author declares that there is no conflict of interest regarding the publication of this article.

\section{Consent}

Informed consent has been obtained from the patient discussed in this case report.

\section{References}

1. Willison HJ, Jacobs BC, van Doorn PA. Guillain-Barre syndrome. Lancet. 2016;388(10045):717-727.

2. Sejvar JJ, Baughman AL, Wise M, Morgan OW. Population incidence of Guillain-Barre syndrome: a systematic review and meta-analysis. Neuroepidemiology. 2011;36(2):123-133.

3. Jacobs BC, Rothbarth PH, van der Meche FG, Herbrink P, Schmitz PI, de Klerk MA, van Doorn PA. The spectrum of antecedent infections in Guillain-Barre syndrome: a case-control study. Neurology. 1998;51(4):1110-1115.

4. Haber P, Sejvar J, Mikaeloff Y, DeStefano F. Vaccines and
Guillain-Barre syndrome. Drug Saf. 2009;32(4):309-323.

5. Schonberger LB, Bregman DJ, Sullivan-Bolyai JZ, Keenlyside RA, Ziegler DW, Retailliau HF, Eddins DL, et al. Guillain-Barre syndrome following vaccination in the National Influenza Immunization Program, United States, 1976--1977. Am J Epidemiol. 1979;110(2):105-123.

6. Lacaille F, Zylberberg H, Hagege H, Roualdes B, Meyrignac C, Chousterman M, Girot R. Hepatitis C associated with Guillain-Barre syndrome. Liver. 1998;18(1):49-51.

7. Stratton K, Ford A, Rusch E, Clayton EW, eds. Adverse Effects of Vaccines: Evidence and Causality. Washington (DC), 2011.

8. Seyal M, Ziegler DK, Couch JR. Recurrent GuillainBarre syndrome following influenza vaccine. Neurology. 1978;28(7):725-726.

9. Marin LF, Abrahao A, Carvalho FA, Santos WA, Dallalba CC, Barcelos LB, Silva GS, et al. Guillain-Barre syndrome associated with H1N1 vaccination. Arq Neuropsiquiatr. 2010;68(6):974-975.

10. Haber P, DeStefano F, Angulo FJ, Iskander J, Shadomy SV, Weintraub E, Chen RT. Guillain-Barre syndrome following influenza vaccination. JAMA. 2004;292(20):2478-2481.

11. Dieleman J, Romio S, Johansen K, Weibel D, Bonhoeffer J, Sturkenboom M, Group V-GC-CS. Guillain-Barre syndrome and adjuvanted pandemic influenza A (H1N1) 2009 vaccine: multinational case-control study in Europe. BMJ. 2011;343:d3908.

12. Kwong JC, Vasa PP, Campitelli MA, Hawken S, Wilson K, Rosella LC, Stukel TA, et al. Risk of Guillain-Barre syndrome after seasonal influenza vaccination and influenza health-care encounters: a self-controlled study. Lancet Infect Dis. 2013;13(9):769-776.

13. Salmon DA, Proschan M, Forshee R, Gargiullo P, Bleser $\mathrm{W}$, Burwen DR, Cunningham F, et al. Association between Guillain-Barre syndrome and influenza A (H1N1) 2009 monovalent inactivated vaccines in the USA: a meta-analysis. Lancet. 2013;381(9876):1461-1468. 